\title{
Migrastatin and Dorrigocins Are Shunt Metabolites of iso-Migrastatin
}

\author{
Jianhua Ju, ${ }^{\#}$ Si-Kyu Lim, ${ }^{\#}$ Hui Jiang,, Ben Shen ${ }^{\#, \&, *}$ \\ ${ }^{\#}$ Division of Pharmaceutical Sciences and ${ }^{\circledR}$ Department of Chemistry, University of Wisconsin- \\ Madison, Madison, Wisconsin 53705, USA \\ Email:bshen@pharmacy.wisc.edu
}

\section{Supporting Information}

General Experimental Procedures. Optical rotations were determined on a Perkin-Elmer 241 instrument at the sodium D line $(589 \mathrm{~nm})$. Electrospray ionization mass spectra (ESI-MS) and LCMS data were obtained on an Agilent 1100 HPLC-MSD SL quadrupole mass spectrometer equipped with both orthogonal pneumatically assisted electrospray and atmospheric pressure chemical ionization sources. High-resolution (HR) MS analyses were acquired on an IonSpec HiResMALDI FT-Mass spectrometer. ${ }^{1} \mathrm{H}$ and ${ }^{13} \mathrm{C}$ NMR spectra were recorded on Varian Unity Inova $500 \mathrm{MHz}$ instruments operating at $500 \mathrm{MHz}$ for ${ }^{1} \mathrm{H}$ and $125 \mathrm{MHz}$ for ${ }^{13} \mathrm{C}$ nuclei. ${ }^{1} \mathrm{H}$ and ${ }^{13} \mathrm{C}$ NMR chemical shifts were referenced to residual solvent signals: $\delta_{\mathrm{H}} 7.27$ and $\delta_{\mathrm{C}} 77.23$ for $\mathrm{CDCl}_{3}$ and $\delta_{\mathrm{H}} 3.31$ and $\delta_{\mathrm{C}}$ 49.15 for $\mathrm{MeOH}-d_{4} \cdot{ }^{1} \mathrm{H}^{-1} \mathrm{H}$ COSY, TOCSY (mixing time for $80 \mathrm{~ms}$ ), HMQC, HMQC-TOCSY, and gHMBC (optimized ${ }^{\mathrm{n}} J_{\mathrm{XH}}$ for $8.0 \mathrm{~Hz}$ ) were performed using standard VARIAN pulse sequences. Silica gel 60A (Fisher Chemical 200-425 mesh) was used for flash column chromatography. Amberlite XAD-16 resin was purchased from Sigma. ${ }^{18} \mathrm{O}$-water $\left({ }^{18} \mathrm{O},>95 \%\right)$ was purchased from Cambridge Isotope Laboratories, Inc.

HPLC was carried out a Varian HPLC system equipped with ProStar 210 pumps and a photodiode detector. Mobile phases used were buffer A (15\% $\mathrm{CH}_{3} \mathrm{CN}$ in $\mathrm{H}_{2} \mathrm{O}$ containing $\left.0.1 \% \mathrm{HOAc}\right)$ and buffer B $\left(80 \% \mathrm{CH}_{3} \mathrm{CN}\right.$ in $\mathrm{H}_{2} \mathrm{O}$ containing $0.1 \% \mathrm{HOAc}$ ). Separation was achieved on a semi-preparative $\mathrm{C} 18$ column (Microsob C18, 250x10 mm, $5 \mu \mathrm{m}$ ), developed with a linear gradient from buffer A/buffer B (88:15) to buffer A/buffer B (10:90) in 20 min and continued at buffer A/buffer B (10:90) for an additional $5 \mathrm{~min}$, at a flow rate of $2.5 \mathrm{ml} / \mathrm{min}$ and monitored by UV detection at $205 \mathrm{~nm}$. Crude fermentation extracts, fractions obtained during isolation, reaction mixture, and the final purified compounds were all analyzed by HPLC (and LC-MS if necessary) on an analytical C18 column (Prodigy ODS-2, 150x4.6 mm, $5 \mu \mathrm{m}$ ), developed with a linear gradient from buffer A/buffer B 
(100:0) to buffer A/buffer B (20:80) in 20 min and continued at buffer A/buffer B (20:80) for an additional $5 \mathrm{~min}$, at flow rate of $1 \mathrm{ml} / \mathrm{min}$ and monitored by UV detection at $205 \mathrm{~nm}$.

Bacterial Strain and Growth Condition. Streptomyces platensis NRRL18993 strain was grown on oatmeal agar plate (prepared by dissolving $3 \mathrm{~g}$ of oatmeal and $2 \mathrm{~g}$ of agar in $100 \mathrm{ml} \mathrm{H}_{2} \mathrm{O}$, boiling the resultant solution for $30 \mathrm{~min}$, and autoclaving for $20 \mathrm{~min})$ at $28^{\circ} \mathrm{C}$ until it well sporulated $(\sim 15$ days $)$. Spores were then harvested and stored in $20 \%$ glycerol at $-80^{\circ} \mathrm{C}$.

Fermentation, Extraction, and Isolation of Compounds 1, 2, 3, and 5. Considerable effort has been devoted to optimize the fermentation conditions of S. platensis NRRL18993 for metabolite production. The condition that produced the most amounts of metabolites was found to be the same as that reported for migrastatin (1) from the Streptomyces sp. MK9929-43F1 strain $^{[1]}$ rather than the one used for 1 production from $S$. platensis $^{[2]}$. A two-stage fermentation procedure was utilized. Seed medium (50 $\mathrm{ml}$ in $250-\mathrm{mL}$ baffled flask) was inoculated with spores, and the flasks were incubated on a rotary shaker at $250 \mathrm{rpm}$ and $28^{\circ} \mathrm{C}$ for 2 days. This seed culture $(50 \mathrm{ml})$ was then transferred into the fermentation medium $(500 \mathrm{ml}$ in 2-L baffled flask), and the flasks were incubated on a rotary shaker at $250 \mathrm{rpm}$ and $28^{\circ} \mathrm{C}$ for 5 days. Both seed and production media consisted of $2 \%$ glycerol, $2 \%$ dextrin, $1 \%$ soytone peptone, $0.3 \%$ yeast extract, $0.2 \%\left(\mathrm{NH}_{4}\right)_{2} \mathrm{SO}_{4}, 0.2 \% \mathrm{CaCO}_{3}, \mathrm{pH}$ 7.0 , and were sterilized by autoclaving at $121^{\circ} \mathrm{C}$ for $30 \mathrm{~min}$.

The production fermentation culture (13 liters) was centrifuged $\left(3,000 \mathrm{rpm}\right.$ at $4^{\circ} \mathrm{C}$ for $\left.16 \mathrm{~min}\right)$ to pellet the mycelia, and the broth was collected and filtrated to afford a clear supernatant. The latter was extracted twice with equal volumes of EtOAc. The combined EtOAc extracts were concentrated under reduced pressure to dryness to yield an oily residue (3.5 g). HPLC and LC-ESI-MS analysis of this crude mixture showed minimally three compounds with a $[\mathrm{M}-\mathrm{H}]^{-}$ion at $\mathrm{m} / z, 506$, corresponding to dorrigocin A (2), B (3) and 13-epi-dorrigocin A (5) and one compound with a [M$\mathrm{H}]^{-}$ion at $m / z 488$, corresponding to $\mathbf{1}$. Compounds $\mathbf{1}, \mathbf{2}, \mathbf{3}$, and $\mathbf{5}$ all showed end UV absorption.

This crude mixture was then subjected to silica gel flash column chromatography $(3.5 \times 40 \mathrm{~cm})$, and the column was developed by stepwise elution with EtOAc-hexane containing 0.1\% HOAc (from 3:7 to 10:0) to EtOAc-MeOH containing 0.1\% HOAc (from 10:0 to 8:2), yielding 25 fractions each of which was analyzed by HPLC. Fractions 9-11 were combined and further purified by silica gel flash column chromatography $\left(1.5 \times 30 \mathrm{~cm}\right.$ ) eluted with $\mathrm{CHCl}_{3}-\mathrm{MeOH}$ (from 10:0 to 9.5:0.5) followed by EtOAC-Hexane (from 4:6 to 10:0) to yield 1 (250 mg or $20 \mathrm{mg} / \mathrm{L}$ ). Fraction 16 was subjected to silica gel flash column chromatography $(1.5 \times 30 \mathrm{~cm})$ eluted with $\mathrm{CHCl}_{3}-\mathrm{MeOH}$ (from 10:0 to 9.5:0.5) to give 3 (180 $\mathrm{mg}$ or $14 \mathrm{mg} / \mathrm{L})$. Fractions $17-19$ were combined and further resolved by semi-preparative HPLC to afford 2 (120 mg or $10 \mathrm{mg} / \mathrm{L})$ and $5(63 \mathrm{mg}$ or $5 \mathrm{mg} / \mathrm{L})$. 
Fermentation, Extraction and Isolation of 4. The fermentation procedure for $\mathbf{4}$ was the same as that for compounds $\mathbf{1}, \mathbf{2}, \mathbf{3}$, and 5 except that the production medium contained $70 \mathrm{~g} / 1 \mathrm{XAD}-16 \mathrm{resin}$, and fermentation time was increased to 10 days. The XAD-16 resins from the production fermentation culture (4 liters) were collected by filtration through gauze sponges and air-dried. The latter was then eluted with anhydrous ethanol and concentrated and concentrated in vacuo. The resultant residue was subjected to silica gel flash column chromatography $(3.5 \times 30 \mathrm{~cm})$ eluted with $\mathrm{CHCl}_{3}-\mathrm{MeOH}$ (from 10:0 to 9.5:0.5). The fraction eluted at $\mathrm{CHCl}_{3}-\mathrm{MeOH}$ (9.7:0.3) was further resolved by repeated silica gel flash column chromatography $(1.5 \times 30 \mathrm{~cm}$ ) developed by EtOAC-Hexane (from 4:6 to $10: 0)$ to yield 4 (320 $\mathrm{mg}$ or $80 \mathrm{mg} / \mathrm{L})$.

\section{Physicochemical Properties of Compounds 1-5.}

Migrastatin (1): Colorless oil; $[\alpha]^{25}+12.5^{\circ}$ (c 3.18, MeOH); ${ }^{1} \mathrm{H}$ NMR, see Table S1; ${ }^{13} \mathrm{C}$ NMR, see Table S2; ESI-MS, [M-H] $]^{-}$ion at $\mathrm{m} / z$ 488.3; HR-MALDI-MS, $[\mathrm{M}+\mathrm{Na}]^{+}$ion at $\mathrm{m} / \mathrm{z} 512.2633$ (calcd for $\left.\left[\mathrm{C}_{27} \mathrm{H}_{39} \mathrm{NO}_{7} \mathrm{Na}\right]^{+}, 512.2624\right)$.

Dorrigocin A (2): Colorless oil; $[\alpha]^{25}+88.5^{\circ}\left(c 0.15\right.$, MeOH); ${ }^{1} \mathrm{H}$ NMR, see Table S1; ${ }^{13} \mathrm{C}$ NMR, see Table S2; ESI-MS, $[\mathrm{M}-\mathrm{H}]^{-}$ion at $\mathrm{m} / z$ 506.3; HR-MALDI-MS, $[\mathrm{M}+\mathrm{Na}]^{+}$ion at $\mathrm{m} / \mathrm{z} 530.2736$ (calcd for $\left[\mathrm{C}_{27} \mathrm{H}_{41} \mathrm{NO}_{8} \mathrm{Na}\right]^{+}, 530.2730$.

Dorrigocin B (3). Colorless oil; $[\alpha]_{\mathrm{D}}^{25}+18.4^{\circ}\left(c\right.$ 0.14, MeOH); ${ }^{1} \mathrm{H}$ NMR, see Table S1; ${ }^{13} \mathrm{C}$ NMR, see Table S2; ESI-MS, $[\mathrm{M}-\mathrm{H}]^{-}$ion at $m / z$ 506.3; HR-MALDI-MS, $[\mathrm{M}+\mathrm{Na}]^{+}$ion at $\mathrm{m} / \mathrm{z} 530.2733$ (calcd for $\left.\left[\mathrm{C}_{27} \mathrm{H}_{41} \mathrm{NO}_{8} \mathrm{Na}\right]^{+,} 530.2730\right)$.

iso-Migrastatin (4). Colorless oil; $[\alpha]^{25}{ }_{\mathrm{D}}+123.9^{\circ}(\mathrm{c} 0.18, \mathrm{MeOH}) ;{ }^{1} \mathrm{H}$ NMR, see Table S1; ${ }^{13} \mathrm{C}$ NMR, see Table S2; ESI-MS, [M-H] ${ }^{-}$ion at $\mathrm{m} / \mathrm{z}, 488.3$; HR-MALDI-MS, $[\mathrm{M}+\mathrm{Na}]^{+}$ion at $\mathrm{m} / \mathrm{z}, 512.2633$ (calcd for $\left[\mathrm{C}_{27} \mathrm{H}_{39} \mathrm{NO}_{7} \mathrm{Na}\right]^{+,}$512.2624).

13-epi-Dorrigocin A (5). Colorless oil; $[\alpha]^{25}{ }_{\mathrm{D}}+14.6^{\circ}(c 0.13, \mathrm{MeOH}) ;{ }^{1} \mathrm{H}$ NMR, see Table S1; ${ }^{13} \mathrm{C}$ NMR, see Table S2; ESI-MS, [M-H] $]^{-}$ion at $\mathrm{m} / z$ 506.3; HR-MALDI-MS, $[\mathrm{M}+\mathrm{Na}]^{+}$ion at $\mathrm{m} / \mathrm{z}$ 530.2736 (calcd for $\left[\mathrm{C}_{27} \mathrm{H}_{41} \mathrm{NO}_{8} \mathrm{Na}\right]^{+}$530.2730).

Large-scale Conversion of 4 to $1,2,3$ and 5 in $\mathrm{H}_{2} \mathrm{O}$. The purity of compound 4 was first analyzed by HPLC showing a single peak (purity $>98 \%$ ) and then confirmed by ${ }^{1} \mathrm{H}$ NMR spectrum (in $\mathrm{CDCl}_{3}$ ) showing no detectable contaminant. A solution of 4 (30 mg in $6 \mathrm{ml}$ of DMSO) was then added to 55 $\mathrm{ml}$ Milli-Q $\mathrm{H}_{2} \mathrm{O}$, and the resultant mixture was incubated at $37^{\circ} \mathrm{C}$ for 2 hours. The reaction mixture was evaporated under high vacuo. The residue was subjected to silica gel flash column 
chromatography eluted with $\mathrm{CHCl}_{3}-\mathrm{MeOH}$ (from 10:0 to 9.4:0.6) to yield pure 1 (8 mg), 3 (9 mg), and a mixture of $\mathbf{2}$ and $\mathbf{5}$. The latter was further resolved by semi-preparative to afford pure $\mathbf{2}$ (6 $\mathrm{mg}$ ) and $5(4 \mathrm{mg})$. The ESI-MS, ${ }^{1} \mathrm{H}$ NMR and optical rotation data of compounds 1, 2, 3, and $\mathbf{5}$ were identical to those obtained from the corresponding compounds isolated from fermentation (the ${ }^{13} \mathrm{C}$ NMR spectrum of $\mathbf{1}$ was also measured, which is identical to that of $\mathbf{1}$ obtained from fermentation).

Conversion of 4 to $1,2,3$ and 5 in $\mathbf{H}_{2}{ }^{18} \mathrm{O}$. In a $1.5 \mathrm{ml}$ microcentrifuge tube, $10 \mu \mathrm{l}$ of 4 (10 $\mathrm{mM}$ in DMSO, purity >98\%) and $90 \mu \mathrm{l}$ of $\mathrm{H}_{2}{ }^{18} \mathrm{O}$ were mixed and incubated at $37^{\circ} \mathrm{C}$ for 2 hours. The resultant reaction mixture was lyophilized, and the residue was dissolved in $0.4 \mathrm{ml}$ of $\mathrm{MeOH}$ for LCMS analysis.

\section{References}

[1] Nakae, K.; Yoshimoto, Y.; Sawa. T.; Homma, Y.; Hamada, M.; Takeuchi, T.; Imoto, M. J. Antibiot. 2000, 53, 1130-1136.

[2] (a) Karwowski, J. P.; Jackson, M.; Sunga, G.; Sheldon, P.; Poddig, J. B.; Kohl, W. L.; Kadan, S. J. Antibiot. 1994, 47, 862-869. (b) Hochlowski, J. E.; Whittern, D. N.; Hill, P.; Mcalpine, J. B. J. Antibiot. 1994, 47, 870-874. (c) Woo, E. J.; Starks, C. M.; Carney, J. R.; Arslanin, R.; Cadapan, L.; Zavala, S.; Licari, P. J. Antibiot. 2002, 55, 141-146. 
Table S1 ${ }^{1} \mathrm{H}$ NMR (500MHz) spectral data of migrastatin (1), dorrigocin A (2) and B (3), isomigrastatin (4), and 13-epi-dorrigocin A (5)

\begin{tabular}{|c|c|c|c|c|c|}
\hline Position & $\mathbf{1}^{a, c}$ & $2^{b, d}$ & $3^{b, d}$ & $\mathbf{4}^{a, c}$ & $5^{b, d}$ \\
\hline 2 & $5.55(16.0,1.5)$ & $5.83 \mathrm{~d}(15.5)$ & $5.85 \mathrm{~d}(15.5)$ & $5.69 \mathrm{~d}(16.0)$ & $5.86 \mathrm{~d}(15.0)$ \\
\hline 3 & $\begin{array}{l}6.47 \text { ddd }(16.0, \\
10.0,3.5)\end{array}$ & $6.96 \mathrm{dt}(15.5,7.0)$ & $6.96 \mathrm{dt}(15.5,6.5)$ & $\begin{array}{l}6.65 \text { ddd }(16.0,8.5, \\
7.5)\end{array}$ & $\begin{array}{l}6.96 \mathrm{dt}(15.0, \\
6.5)\end{array}$ \\
\hline 4 & $2.20 \mathrm{~m}, 2.41 \mathrm{~m}$ & $2.36,2 \mathrm{H}, \mathrm{m}$ & $2.38,2 \mathrm{H}, \mathrm{m}$ & $2.15 \mathrm{~m} ; 2.45 \mathrm{~m}$ & $2.37,2 \mathrm{H}, \mathrm{m}$ \\
\hline 5 & $2.20 \mathrm{~m}, 2.41 \mathrm{~m}$ & $2.30,2 \mathrm{H}, \mathrm{m}$ & $2.32,2 \mathrm{H}, \mathrm{m}$ & $1.96 \mathrm{~m}, 2.60 \mathrm{~m}$ & $2.31,2 \mathrm{H}, \mathrm{m}$ \\
\hline 6 & $\begin{array}{l}5.50 \text { ddd }(15.5 \\
10.0,5.0)\end{array}$ & $5.73 \mathrm{dt}(15.5,6.5)$ & $5.79 \mathrm{dt}(15.5,6.5)$ & $\begin{array}{l}5.60 \text { ddd }(15.5, \\
11.0,4.5)\end{array}$ & $\begin{array}{l}5.76 \mathrm{dt}(15.5, \\
6.5)\end{array}$ \\
\hline 7 & $5.22 \mathrm{dd}(15.5,5.0)$ & $\begin{array}{l}5.51 \text { brdd }(16.0, \\
8.5)\end{array}$ & 5.25 brdd $(15.5,8.5)$ & $5.10(15.5,3.5)$ & $\begin{array}{l}5.50 \mathrm{dd}(15.5, \\
8.5)\end{array}$ \\
\hline 8 & $3.46 \mathrm{dd}(8.5,5.0)$ & $3.55 \mathrm{dd}(8.5,4.0)$ & $3.50 \mathrm{dd}(8.5,4.0)$ & $3.43 \mathrm{~m}$ & $3.46 \mathrm{dd}(8.5,4.0)$ \\
\hline 9 & $3.02 \mathrm{dd}(8.5,1.5)$ & $3.20 \mathrm{dd}(7.0,4.0)$ & $3.36 \mathrm{dd}(8.5,2.0)$ & $3.75(9.5)$ & $3.13 \mathrm{dd}(7.5,4.0)$ \\
\hline 10 & $2.91 \mathrm{~m}$ & $2.72 \mathrm{dq}(10.0,7.0)$ & $1.75 \mathrm{~m}$ & $1.85 \mathrm{~m}$ & $\begin{array}{l}2.71 \mathrm{dq}(10.0, \\
7.0)\end{array}$ \\
\hline 11 & $5.62 \mathrm{dd}(10.5,1.5)$ & $5.29 \mathrm{~d}(10.0)$ & $3.99 \mathrm{~d}(8.5)$ & 5.20 (overlap) & $5.30 \mathrm{~d}(10.0)$ \\
\hline 13 & $5.06 \mathrm{~d}(10.0)$ & $4.01 \mathrm{~d}(10.0)$ & $5.29 \mathrm{~d}(10.0)$ & 5.20 (overlap) & $4.20 \mathrm{~d}(7.0)$ \\
\hline 14 & $2.95 \mathrm{dd}(10.0,8.5)$ & $2.78 \mathrm{dq}(10.0,7.0)$ & $3.53 \mathrm{~m}$ & $3.43 \mathrm{~m}$ & 2.81 quintet (7.0) \\
\hline 16 & $2.48,2 \mathrm{H}, \mathrm{t}(7.0)$ & $2.63,2 \mathrm{H}, \mathrm{m}$ & $2.51,2 \mathrm{H}, \mathrm{m}$ & $2.39 \mathrm{~m} ; 2.62 \mathrm{~m}$ & $2.58,2 \mathrm{H}, \mathrm{m}$ \\
\hline 17 & $1.59,2 \mathrm{H}, \mathrm{m}$ & $1.62,2 \mathrm{H}, \mathrm{m}$ & $1.59,2 \mathrm{H}, \mathrm{m}$ & $1.59,2 \mathrm{H}, \mathrm{m}$ & $1.59,2 \mathrm{H}, \mathrm{m}$ \\
\hline 18 & $1.32,2 \mathrm{H}, \mathrm{m}$ & $1.39,2 \mathrm{H}, \mathrm{m}$ & $1.37,2 \mathrm{H}, \mathrm{m}$ & $1.36,2 \mathrm{H}, \mathrm{m}$ & $1.37,2 \mathrm{H}, \mathrm{m}$ \\
\hline 19 & $2.10 \mathrm{~m}$ & $2.14 \mathrm{~m}$ & $2.13 \mathrm{~m}$ & $2.10 \mathrm{~m}$ & $2.12 \mathrm{~m}$ \\
\hline 20 & $2.20 \mathrm{~m} ; 2.66 \mathrm{~m}$ & $2.31 \mathrm{~m} ; 2.66 \mathrm{~m}$ & $2.32 \mathrm{~m} ; 2.64 \mathrm{~m}$ & $2.25 \mathrm{~m} ; 2.70 \mathrm{~m}$ & $2.31 \mathrm{~m} ; 2.64 \mathrm{~m}$ \\
\hline 22 & $0.94 \mathrm{~d}(7.0)$ & $0.97 \mathrm{~d}(6.5)$ & $0.93 \mathrm{~d}(7.0)$ & $0.87(7.5)$ & $0.98 \mathrm{~d}(6.5)$ \\
\hline 23 & $1.83,3 \mathrm{H}, \mathrm{d}(1.5)$ & $1.64 \mathrm{~d}(1.0)$ & $1.67 \mathrm{~d}(1.0)$ & $1.90(1.5)$ & $1.64 \mathrm{~d}(1.0)$ \\
\hline 24 & $1.10,3 \mathrm{H}, \mathrm{d}(8.5)$ & $0.83 \mathrm{~d}(7.0)$ & $1.13 \mathrm{~d}(7.0)$ & $1.14(7.0)$ & $1.09 \mathrm{~d}(7.0)$ \\
\hline 25 & $2.20 \mathrm{~m} ; 2.66 \mathrm{~m}$ & $2.31 \mathrm{~m} ; 2.66 \mathrm{~m}$ & $2.32 \mathrm{~m} ; 2.64 \mathrm{~m}$ & $2.25 \mathrm{~m} ; 2.70 \mathrm{~m}$ & $2.31 \mathrm{~m} ; 2.64 \mathrm{~m}$ \\
\hline $8-\mathrm{OCH}_{3}$ & $3.28,3 \mathrm{H}, \mathrm{s}$ & $3.22 \mathrm{~s}$ & $3.26 \mathrm{~s}$ & $3.34 \mathrm{~s}$ & $3.20 \mathrm{~s}$ \\
\hline
\end{tabular}

${ }^{\mathrm{a}}$ Signals were assigned with aid of ${ }^{1} \mathrm{H}-{ }^{1} \mathrm{H}$ COSY and HMQC experiments. ${ }^{\mathrm{b}}$ Signals were assigned with aid of ${ }^{1} \mathrm{H}-{ }^{1} \mathrm{H}$ COSY, TOCSY, HMQC, HMQC-TOCSY, and gHMBC experiments. $\quad{ }^{\text {SSample }}$ measured in $\mathrm{CDCl}_{3} \cdot{ }^{\mathrm{d}}$ Sample measured in $\mathrm{CD}_{3} \mathrm{OD}$. 
Table S2 ${ }^{13}$ C NMR (125 MHz) spectral data of migraststin (1) dorrigocin A (2) and B (3), isomigraststin (4), and 13-epi-dorrigocin A (5)

\begin{tabular}{|c|c|c|c|c|c|}
\hline$\overline{\text { Position }}$ & $1^{a, c}$ & $2^{b, d}$ & $\mathbf{3}^{b, d}$ & $4^{a, c}$ & $5^{b, d}$ \\
\hline 1 & 164.0 & 170.1 & 170.0 & 167.8 & $170.0^{e}$ \\
\hline 2 & 122.3 & 123.4 & 123.3 & 125.2 & $123.8^{e}$ \\
\hline 3 & 150.1 & 150.2 & 150.2 & 150.8 & 150.3 \\
\hline 4 & 30.5 & 32.8 & 32.8 & $30.3^{*}$ & 32.9 \\
\hline 5 & 31.2 & 32.0 & 32.0 & 32.9 & 32.0 \\
\hline 6 & 130.6 & 135.3 & 136.6 & 129.3 & 135.3 \\
\hline 7 & 128.1 & 130.4 & 129.1 & 130.5 & 130.5 \\
\hline 8 & 82.7 & 84.5 & 86.3 & 81.8 & 84.4 \\
\hline 9 & $\overline{78.0}$ & 79.1 & 75.8 & 73.4 & 79.2 \\
\hline 10 & 32.1 & 36.0 & 38.3 & 38.3 & 35.9 \\
\hline 11 & 133.2 & 133.7 & 80.7 & 82.4 & 131.6 \\
\hline 12 & 131.3 & 136.0 & 140.3 & 134.3 & 136.3 \\
\hline 13 & 77.1 & 82.0 & 128.0 & 128.2 & 78.6 \\
\hline 14 & 51.2 & 50.4 & 47.3 & 46.2 & 51.5 \\
\hline 15 & 211.0 & 216.7 & 213.9 & 211.0 & 215.0 \\
\hline 16 & 40.3 & 43.8 & 41.5 & 40.1 & 42.1 \\
\hline 17 & 20.3 & 21.2 & 21.7 & 20.5 & 21.6 \\
\hline 18 & 34.3 & 35.3 & 35.4 & 34.4 & 35.4 \\
\hline 19 & 30.2 & 31.6 & 31.6 & $30.3^{*}$ & 31.8 \\
\hline 20,25 & $37.8 *$ & 38.7 & 38.6 & 37.9 & 38.7 \\
\hline 21,26 & 172.5 & 175.7 & 175.7 & 172.3 & 175.6 \\
\hline 22 & 13.5 & 16.5 & 8.6 & 10.7 & 16.4 \\
\hline 23 & 26.1 & 10.9 & 12.4 & 13.5 & 13.0 \\
\hline 24 & 13.5 & 14.0 & 16.6 & 15.9 & 12.4 \\
\hline $8-\mathrm{OCH}_{3}$ & 57.0 & 56.5 & 56.7 & 57.3 & 56.5 \\
\hline
\end{tabular}


${ }^{a}$ Signals were assigned with aid of ${ }^{1} \mathrm{H}-{ }^{1} \mathrm{H}$ COSY and HMQC experiments. ${ }^{b}$ Signals were assigned with aid of ${ }^{1} \mathrm{H}-{ }^{1} \mathrm{H}$ COSY, TOCSY, HMQC, HMQC-TOCSY, and $g \mathrm{HMBC}$ experiments. ${ }^{c}$ Samples measured in $\mathrm{CDCl}_{3} .{ }^{d}$ Samples measured in $\mathrm{CD}_{3} \mathrm{OD}$. ${ }^{e}$ Not observed in ${ }^{13} \mathrm{C}$ NMR spectrum but observed as a cross-peak in HMQC or $g \mathrm{HMBC}$ spectra. *Overlapped signals. 